\title{
Predictive Value of Dosimetric Parameters and Absolute Monocyte Count (AMC) for Acute Hematologic Toxicity in Cervical Cancer Patients Undergoing Concurrent Chemotherapy and Volumetric-Modulated Arc Therapy (VMAT)
}

Xiaoyong Xiang ( $\nabla$ xiangxiaoyong16@163.com )

National cancer center/national clinical research center for cancer/cancer hospital \& Shenzhen hospital, chinese academy of medical sciences and peking union medical college, Shenzhen, 518116,Chian

\section{Zhen Ding}

Cancer hospital \& Shenzhen Hospital

Qi Zeng

Cancer Hospital \& Shenzhen Hospital

Lingling Feng

Cancer Hospital \& Shenzhen Hospital

Chunyan Qiu

Cancer hospital \& Shenzhen Hospital

Dongjie Chen

Cancer Hospital \& Shenzhen Hospital

Jiawei Lu

Cancer Hospital \& Shenzhen Hospital

Ning Li

Cancer Hospital \& Shenzhen Hospital

\section{Research}

Keywords: bone marrow, acute hematological toxicity, cervical cancer, volumetric-modulated arc therapy (VMAT), absolute monocyte count (AMC)

Posted Date: October 18th, 2021

DOI: https://doi.org/10.21203/rs.3.rs-966809/v1

License: (9) This work is licensed under a Creative Commons Attribution 4.0 International License. Read Full License 


\section{Abstract}

Purpose: To identify clinical/dosimetric predictors of acute hematologic toxicity (HT) in cervical cancer patients undergoing concurrent chemotherapy and volumetric-modulated arc therapy (VMAT).

Methods and Materials: We retrospectively analyzed 184 cervical cancer patients receiving concurrent chemotherapy and VMAT. Hematological parameters were collected during the treatment period. The total pelvic bone(TPB) was retrospectively delineated for each patient, and the volume of TPB receiving 10,20 , 30,40 , and 50 Gy (V10, V20, V30, V40, and V50, respectively) was calculated. We assessed the correlations between variables by the Spearman rank correlation test and compared the differences between groups by the Wilcoxon signed-rank test. Binary logistic regression analysis was used to analyze associations between $\mathrm{HT}$ and clinical/dosimetric parameters. The receiver operating characteristic curve(ROC) was used to determine the best cut-off values for dosimetric planning constraints.

Results: The nadir of absolute monocyte count (AMC) was positively correlated with the nadir of absolute white blood cells (WBC) count $(r \otimes 0.5378,95 \% \mathrm{Cl} \otimes 0.4227$ to $0.6357, \mathrm{P}<0.0001)$ and the nadir of absolute neutrophil count(ANC) $(r \otimes 0.5000 \otimes 95 \% \mathrm{Cl} \otimes 0.3794$ to $0.6039, \mathrm{P}<0.0001)$. The decrease and increase of AMC usually occurred before the ANC and WBC. In multivariate logistic regression analysis, the chemotherapy regimen and the TPB_V20 were independent risk factors for developing Grade $\geq 3$ hematologic toxicity. The optimal TPB_V20 cut-off value identified by ROC curves followed by Youden

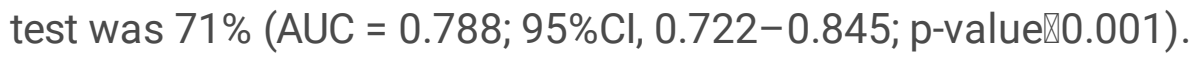

Conclusions: The changing trend of $\mathrm{AMC}$ can be used as an effective predictor for the timing and severity of the ANC/WBC nadirs. Maintain TPB_V20 $<71 \%$ and selecting single-agent cisplatin or carboplatin could significantly reduce Grade $\geq 3 \mathrm{HT}$ in cervical cancer patients undergoing concurrent chemoradiotherapy.

\section{Introduction}

According to the global cancer statistics released in 2020, cervical cancer (CC) is one of the most common cancers of the reproductive system in women and is responsible for $\sim 340,000$ annual mortalities ${ }^{[1]}$. Radical surgery is still the first treatment strategy for early-stage cervical cancer. However, for patients with high-risk surgical-pathological factors (such as pelvic lymph node-positive, positive resection margins, and parametrial infiltration) or locally advanced cervical cancer, concurrent platinumbased chemoradiotherapy remains one of the main therapeutic options ${ }^{[2]}$. Previous studies suggested that concurrent chemoradiotherapy (CCRT) can improve treatment efficacy and increases adverse reactions, and one of the main adverse effects is hematological toxicity $(\mathrm{HT})$ during the CCRT and followup $^{[3,4]}$.

Patients with grade 3 or greater HT are more likely to develop life-threatening infections or febrile neutropenia,which could reduce CCRT doses or termination of CCRT altogether $\square$ and ultimately affect the 
therapeutic efficacy ${ }^{[5]}$. Therefore, in addition to further effectively reducing the severity and incidence of hematological toxicity, improving adequate pretreatment evaluation of leukopenia and neutropenic risk may help ensure that most patients continue to receive the optimal dose intensity chemoradiotherapy, thus making them more likely to achieve their treatment goals.

In cancer patients, approximately $34.5 \%$ of the active bone marrow is located within the pelvis bones, $16.6 \%$ within the lumbar vertebrae, and $4.5 \%$ within the proximal femur ${ }^{[6]}$. Owing to the high radiosensitivity of hematopoietic stem cells, the pelvic bone is a potential organ at risk, especially in patients with cervical cancer receiving CCRT. In addition, we have observed clinically that the decrease and increase of absolute monocyte count (AMC) occurred before the absolute neutrophil count(ANC)/absolute white blood cells (WBC) count, and there is a clear correlation between the nadir of AMC and the severity of neutropenia/leukopenia.

Currently, advanced treatment techniques, including intensity-modulated radiation therapy (IMRT), helical tomography radiotherapy (TOMO), or volumetric-modulated arc therapy (VMAT) regimens, have been used to treat cervical cancer. A recent dosimetric study has shown that the use of VMAT with os coxae and lumbosacral spine as separate dose-volume constraints in patients with cervical cancer can reduce $\mathrm{HT}$ associated with $\mathrm{CCRT}^{[7]}$. However, no uniform standard for limiting the optimal dosimetric parameters of pelvic bones marrow to reduce the incidence of severe $\mathrm{HT}$ is available yet ${ }^{[8,9]}$. Therefore, the aim of our study is to explore the clinical and dosimetric predictors of HT in cervical cancer patients treated with CCRT in our institution. We also examined the predictive value of AMC for acute leukopenia/neutropenia.

\section{Methods And Materials}

\section{Patients}

We retrospectively reviewed the clinical data of 184 patients with cervical cancer undergoing CCRT at our institution between October 2018 to March 2021. All the eligible patients had newly diagnosed, or recurrent cervical cancer confirmed by biopsy and received either definitive or postoperative pelvic radiotherapy with concurrent weekly platinum-based chemotherapy. The pelvic radiotherapy was administered with an image-guided VMAT technique. Patients who received conventional 3D-CRT or IMRT and patients without complete clinical data were excluded from our study. Except for anemia, patients with grade $\geq 2$ other hematologic toxicity in the last two weeks before the concurrent chemoradiotherapy and patients with known bone marrow metastasis were also excluded. During the chemoradiotherapy period, the patients were assessed for hematologic toxicity at least once a week by complete blood counts. All patients in the study signed informed consent forms.

\section{Concurrent chemotherapy}

All chemotherapy regimens were platinum-based. The chemotherapy regimens included TP (135-175 $\mathrm{mg} / \mathrm{m}^{2}$ paclitaxel, D1; 50-70 mg/ $\mathrm{m}^{2}$ cisplatin, D2-4; 21 day repeat, 1-2 cycles) $\llbracket$ weekly cisplatin $₫ 40 \mathrm{mg} / \mathrm{m}^{2}$, D1, 5-6 cycles) and weekly carboplatin (AUC2, D1, 5-6 cycles). Before each cycle of chemotherapy, all 
patients underwent routine hematological examination, including hematology, blood chemistry, renal function, liver function. Patients have received the next cycle of chemotherapy if they tolerance to the previous cycle.

\section{Volumetric-modulated arc therapy}

Typically, the patient was simulated in the prone position. If necessary, the patient's immobilization was performed in the supine position. A contrast-enhanced CT-based simulation was performed with $5 \mathrm{~mm}$ slice-thickness when patients were moderately filling the bladder and emptying the rectum. The coverage of CT scan was from the lower edge of the T10 vertebrae to the $5 \mathrm{~cm}$ below the ischial tuberosity. If there were metastatic lesions near the abdominal aorta, the coverage of CT scan should also include an extratumor $10 \mathrm{~cm}$.

The clinical target area (CTV) included the entire cervix囚uterus, partial vaginal, vesicovaginal, rectovaginal spaces, gross tumor, parametria, regional lymph nodes. Lymph node metastasis was defined by imaging

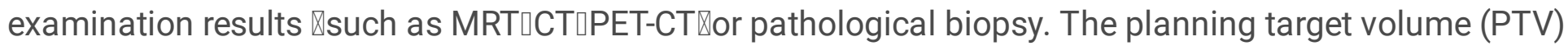
was included the CTV and a uniform three-dimensional expansion of $5 \mathrm{~mm}$.

In this study, all the patients underwent pelvic volumetric-modulated arc therapy. The pelvic radiation dose was 45-50.4 Gy in 1.8Gy daily fractions, and the dose delivered to the involved lymph nodes was 58.8-50.2 Gy in 2.1-2.15Gy daily fractions. The following OARs(organs at risk) were delineated for dosevolume calculations: bladder, spinal cord, femoral heads, rectum, small intestine, sigmoid colon, and bowel bag. The pelvic bone marrow was not included in the OARs but was delineated for dose-volume calculations retrospectively. The cone-beam computed tomography was performed to minimize the daily setup error. The vaginal brachytherapy was applied based on the patients' specific circumstances, and the dose to pelvic bone marrow from brachytherapy was considered barely relevant.

\section{Bone marrow delineation}

All bone marrow contours were retrospectively delineated manually according to Mell et al ${ }^{[9]}$. To ensure repeatability and limit inter-observer variations, all the external contours of the bones within the PTV coverage (defined as from the centrum $2 \mathrm{~cm}$ above the upper boundary of PTV to the ischial tuberosities) were retrospectively delineated as a proxy for the bone marrow rather than the low-density areas within the bones by a single radiation oncologist and subsequently reviewed by another senior radiation oncologist. The contour of the total pelvic bone(TPB) was shown in Figure 1.

Dose-volume histograms (DVHs) corresponding to the delivered VMAT plan were used to assess the dose volumes(V10, V20, V30, V40, V50, respectively) received by the TPB.

\section{Hematologic toxicity}

All patients had accepted blood routine examination before receiving external irradiation or each platinum-based chemotherapy. During the period of CCRT and within two weeks following external 
radiation therapy completion, the blood routine examination should be taken at least once a week, and the frequency of blood routine examination could be increased if necessary. The day of CCRT initiation was defined as day 0 (d1), and the first day after CCRT initiation was defined as day 1 (d1). When AMC, ANC, PLT, WBC, and HGB were reduced to a minimal level, the days from D0 were calculated, respectively. According to a similar approach, when AMC, ANC, and WBC increased from the nadir to $\geq$ normal value for the first time, the days from the $\mathrm{d} 1$ were calculated, respectively.

The HT was graded according to the Common Terminology Criteria for Adverse Events (CTCAE) v5.0. Whether the patients were treated with granulocyte-monocyte colony-stimulating factors, red cell transfusion, or platelet transfusion was based on attending physicians' decisions according to clinical judgment.

\section{Statistical analysis}

Data analysis was performed using SPSS (IBM SPSS 23.0, SPSS Inc). Descriptive statistics were generated for relevant clinical and dosimetric parameters. The Shapiro-Wilk test checked the normality of data distribution. Those conforming to normal distribution were expressed as mean \pm standard deviation. Those not conforming to normal distribution were expressed as the median and interquartile range (IQR). Categorical data were assessed and described as frequencies and percentages. According to the normality test results, paired-samples t-test or Wilcoxon signed-rank test was performed to compare the differences between two groups. Pearson's correlation analysis or Spearman's correlation was used to testing the correlation between the two variables, with $p<0.05$ considered significant. Possible risk factors of Grade $\geq 3$ hematologic toxicity were analyzed with binary logistic regression analysis, then variable with P-value $<0.05$ in the binary logistic regression analysis were put into the multiple logistic regression analysis. The receiver operating characteristic (ROC) curve was used to evaluate the area under the ROC curve (AUC), best cut-off point, specificities, and sensitivities. A $p<0.05$ was considered statistically significant.

\section{Results}

\section{Patient characteristics}

We retrospectively reviewed the clinical data of 184 patients with cervical cancer undergoing CCRT in our institution between October 2018 to March 2021. All patients received pelvic radiotherapy with an imageguided VMAT technique. All patients were treated with platinum-based chemotherapy; 42 (22.8\%) received TP, $111(60.3 \%)$ received weekly cisplatin, and 31 (16.9\%) received weekly carboplatin. All patients received at least one cycle of concurrent chemotherapy (with a median of 2 cycles, range from 1 to 6 cycles). Basic clinical characteristics of the patients are available in Table 1.

Table 1. Basic clinical characteristics of the patients. 


\begin{tabular}{|c|c|c|}
\hline Patients (n) & & 184 \\
\hline Age (years) & Median, Mean (Range, SD) & $54,53.3(31-81,10.3)$ \\
\hline BMI (『/ه) & Median, Mean (Range, SD) & $22.59,23.1(16.2-33.8,3.3)$ \\
\hline Duration of EBRT (days) & Median, Mean (Range, SD) & $37,38(29-52,3.9)$ \\
\hline RT dose to pelvis (Gy) & Median, Mean (Range, SD) & $45,47.4 \llbracket 45-50.4,2.6 \rrbracket$ \\
\hline Cycles of chemotherapy & Median, Mean (Range, SD) & $2,2.9 \unrhd 1-6,1.5 \rrbracket$ \\
\hline \multirow[t]{2}{*}{ Clinical stage FIGO2018 (n, \%) } & IB-IIB & $98 \rrbracket 53.3 \rrbracket$ \\
\hline & IIIA-IVB & 86ه46.7区 \\
\hline \multirow[t]{2}{*}{ Histology, n (\%) } & Squamous carcinoma & $166 \rrbracket 90.2 \rrbracket$ \\
\hline & Adenocarcinoma & $18 \varangle 9.8 \rrbracket$ \\
\hline \multirow[t]{2}{*}{ Differentiation degree, n (\%) } & High-moderate & $85 \rrbracket 46.2 \rrbracket$ \\
\hline & Lower / Unknown & $99 \bowtie 53.8 \rrbracket$ \\
\hline PTV dose-pelvis & 45 Gy & $99 \otimes 53.8 \rrbracket$ \\
\hline$(n, \%)$ & 48.6Gy-50.4 Gy & $85 \rrbracket 46.2 \rrbracket$ \\
\hline \multirow[t]{3}{*}{ Chemotherapy regimen $(n, \%)$} & TP & $42 \rrbracket 22.8 \rrbracket$ \\
\hline & Carboplatin & $31 \otimes 16.9 \bigotimes$ \\
\hline & Cisplatin & 111ه60.3凶 \\
\hline
\end{tabular}

Abbreviation: BMI, body mass index; EBRT, pelvic external-beam radiotherapy; SD, standard deviation; RT, radiation therapy; TP, paclitaxel + cisplatin.

\section{Dosimetric parameters of the pelvic bone}

Descriptive statistical analysis of the dosimetric parameters was performed, and their mean values, median values, maximum, minimum, 25th percentile, 75th percentile, and standard deviations were recorded. Table 2 details the dosimetric parameters for the total pelvic bone marrow.

Table 2. Descriptive statistics of dosimetric parameters of the total pelvic bones. 


\begin{tabular}{|lllllll|}
\hline Parameter & & Mean & Median & Min-max & Q1-Q3 & SD \\
\hline TPB & Volume $\left(\mathrm{cm}^{3}\right)$ & 1189 & 1165 & $886-2219$ & $1070-1266$ & 177.6 \\
& V10 (10\%) & 90.39 & 90.00 & $49.00-100.00$ & $88.00-94.00$ & 5.780 \\
& V20(20\%) & 71.53 & 70.74 & $27.23-92.90$ & $66.95-76.89$ & 7.977 \\
& V30 (30\%) & 45.41 & 45.00 & $14.00-68.00$ & $39.00-51.00$ & 8.832 \\
& V40 (40\%) & 23.32 & 23.00 & $6.00-46.00$ & $18.00-27.00$ & 6.617 \\
& V50(50\%) & 5.14 & 3.00 & $0.00-26.00$ & $0.00-10.00$ & 5.422 \\
\hline
\end{tabular}

Abbreviation: V10, V20, V30, V40, V50囚volume receiving 10,20, 30, 40, 50 Gy; TPB, the total pelvic bones; Max, maximum; Min, minimum; Q1, 25th percentile; Q3, 75th percentile; SD, standard deviation.

\section{Hematological baselines and nadirs}

Compared with the hematological baselines, all the blood cell counts were decreased to various degrees throughout the study period $(P \otimes 0.001)$. The median of WBC, ANC, HGB, AMC, and PLT counts at the nadirs

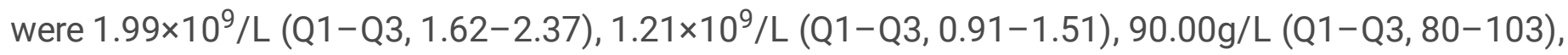
$0.21 \times 10^{9} / \mathrm{L}(\mathrm{Q} 1-\mathrm{Q} 3,0.14-0.28)$, and $104.0010^{9} / \mathrm{L}(\mathrm{Q} 1-\mathrm{Q} 3,78.25-131.00)$, respectively. The WBC, ANC, HGB, AMC and PLT counts decreased by $64.72 \%, 64.86 \%, 16.00 \%, 50.00 \%, 53.44 \%$, respectively. (Figure 2).

\section{Hematologic toxicity}

At baseline, 26 patients (14.13\%) had Grade 1 leukopenia, 11 patients(5.98\%) had Grade 1 neutropenia, and 2 patients(1.09\%) had Grade 1 thrombocytopenia. Moreover, anemia was more common at baseline, compared to other Hematologic toxicity. At baseline, 50 patients(27.17\%) had Grade 1, 20 patients(10.89\%) had Grade 2, and 7 patients(3.08\%) had Grade 3 anemia. The percentage of Grade 3 or Grade 4 leukopenia, neutropenia, thrombocytopenia, and anemia during concurrent chemoradiotherapy was $52.17 \%, 30.43 \%, 6.52 \%$, and $22.83 \%$, respectively. Overall, 111 patients $₫ 60.33 \% \bigotimes$ did experience any Grade 3+ hematologic toxicity.

Detailed data are given in Table 3.

Table 3. Hematological toxicity Graded according to hematological nadirs and baselines. 


\begin{tabular}{|c|c|c|c|c|c|c|}
\hline \multicolumn{2}{|c|}{ Hematologic toxicity } & Grade 0 & Grade 1 & Grade 2 & Grade 3 & Grade 4 \\
\hline & & & & & & \\
\hline \multirow[t]{4}{*}{ Baselines } & Leukopenia & 158囚85.87囚 & $26 \rrbracket 14.13 \rrbracket$ & $0 \otimes 0 \rrbracket$ & $0 \otimes 0 \rrbracket$ & $0 \otimes 0 \rrbracket$ \\
\hline & Neutropenia & 173ه94.02》 & $11 \rrbracket 5.98 \rrbracket$ & $0 \otimes 0 \rrbracket$ & $0 \otimes 0 \otimes$ & $0 \otimes 0 \bigotimes$ \\
\hline & Thrombocytopenia & 182ه98.91囚 & $2 \otimes 1.09 \rrbracket$ & $0 \otimes 0 \rrbracket$ & $0 \otimes 0 \otimes$ & $0 \otimes 0 \rrbracket$ \\
\hline & Anemia & 107ه58.15】 & $50 \rrbracket 27.17 \rrbracket$ & 20ه10.89ه & 7邓3.80】 & $0 \otimes 0 \bigotimes$ \\
\hline \multirow[t]{4}{*}{ Nadirs } & Leukopenia & $0 \otimes 0 \rrbracket$ & $9 \rrbracket 4.89 \rrbracket$ & $79 \llbracket 42.93 \rrbracket$ & 88\47.83》 & $8 \rrbracket 4.35 \rrbracket$ \\
\hline & Neutropenia & $9 \rrbracket 4.89 \rrbracket$ & 41区22.28ه & $78 \rrbracket 42.39 \rrbracket$ & $46 \rrbracket 25.00 \rrbracket$ & $10 \rrbracket 5.43 \rrbracket$ \\
\hline & Thrombocytopenia & 100冈53.8ه & $45 \rrbracket 24.46 \rrbracket$ & 27囚14.67囚 & $11 \rrbracket 5.98 \rrbracket$ & 1ه0.54囚 \\
\hline & Anemia & 17凶9.24】 & $62 \rrbracket 33.70 \rrbracket$ & $63 \rrbracket 34.24 \rrbracket$ & 29ه15.76ه & 13ه7.07ه \\
\hline
\end{tabular}

\section{The changing trend of blood cell counts}

The median time to the nadir was 26 days (Q1-Q3, 19 to 34 days) for the WBC and ANC, 20 days (Q1Q3, 8 to 29 days) for the AMC, 29 days (Q1-Q3, 20 to 39 days) for the PLT, 35 days (Q1-Q3, 24 to 42 days) for the HGB. Compared with the WBC and ANC, the AMC was decreased to the nadir first (median = 5 days, $\mathrm{Q} 1-\mathrm{Q} 3=0$ to 11 days $\triangle \mathrm{P}<0.001$ ). (Figure 3. A and Figure 3. B). The time for AMC to increase from the nadir to $\geq$ normal value for the first time was less than that for WBC (median $=4$ days, Q1 $-Q 3=0$ to 11 days $\triangle \mathrm{P}<0.001$ ) and $\mathrm{ANC}$ (median $=3$ days, $\mathrm{Q} 1-\mathrm{Q} 3=0$ to 10 days $\triangle \mathrm{P}<0.001$ ). (Figure 3 . $\mathrm{C}$ and Figure 3 . D ). We also assessed the correlations between variables by the Spearman rank correlation test. We obtained that the nadir of AMC was positively correlated with the nadir of WBC $(\mathrm{r} \otimes 0.5378,95 \% \mathrm{CI} \otimes 0.4227$ to $0.6357, \mathrm{P}<0.0001)$ and the nadir of $\mathrm{ANC}(\mathrm{r} \otimes 0.5000 \otimes 95 \% \mathrm{Cl} \otimes 0.3794$ to $0.6039, \mathrm{P}<0.0001)$. (Figure 3 . $\mathrm{E}$ and Figure 3. F).

All analyses above indicate that the decrease and increase of AMC usually occurred before the ANC and WBC $\triangle$ and the changing tendency of $A M C$ had some predictive value to the changing trend of WBC and ANC.

\section{Predictors of hematologic toxicity}

Theunivariate and multiple binary logistic regression analyses were performed to identify any Grade $\geq 3$ $\mathrm{HT}$ predictors. The chemotherapy regimen and the total pelvic bone(TPB_V10, TPB_V20, TPB_V30, TPB_V40, and TPB_V50) were in univariable binary logistic regression analysis associated with any Grade $\geq 3$ HT (Table 4). 
Table 4 Univariate logistic regression analysis of factors associated with the development of any Grade $\geq 3$ hematologic toxicity.

\begin{tabular}{|c|c|c|c|c|}
\hline \multicolumn{2}{|l|}{ Parameter } & Odds ratio & $95 \% \mathrm{Cl}$ & $P$-value \\
\hline \multicolumn{2}{|l|}{ Age (years) } & 1.000 & $0.972-1.029$ & 0.991 \\
\hline \multicolumn{2}{|l|}{ BMI (『/囚) } & 0.982 & $0.898-1.074$ & 0.982 \\
\hline \multicolumn{2}{|l|}{ Duration of EBRT (days) } & 1.069 & $0.987-1.158$ & 0.102 \\
\hline \multicolumn{2}{|l|}{ Clinical stage (FIGO2018) } & 1.759 & $0.964-3.208$ & 0.066 \\
\hline \multicolumn{2}{|l|}{ Histology } & 0.559 & $0.232-1.345$ & 0.194 \\
\hline \multicolumn{2}{|l|}{ Differentiation degree } & 0.789 & $0.431-1.445$ & 0.443 \\
\hline \multicolumn{2}{|l|}{ PTV dose-pelvis } & 1.696 & $0.930-3.094$ & 0.085 \\
\hline \multicolumn{2}{|l|}{ Cycles of chemotherapy } & 1.056 & $0.861-1.295$ & 0.600 \\
\hline \multicolumn{2}{|l|}{ Volume $\left(\mathrm{cm}^{3}\right)$} & 1.002 & $1.000-1.004$ & 0.053 \\
\hline \multirow{3}{*}{ Chemotherapy regimen } & TP & & & $0.027 *$ \\
\hline & Carboplatin & 0.257 & $0.094-0.701$ & $0.008 *$ \\
\hline & Cisplatin & 0.442 & $0.198-0.987$ & $0.046 *$ \\
\hline \multicolumn{2}{|l|}{ TPB_V10 } & 1.197 & $1.109-1.292$ & [0.001* \\
\hline \multicolumn{2}{|l|}{ TPB_V20 } & 1.176 & $1.109-1.248$ & [0.001* \\
\hline \multicolumn{2}{|l|}{ TPB_V30 } & 1.102 & $1.057-1.149$ & [0.001* \\
\hline \multicolumn{2}{|l|}{ TPB_V40 } & 1.114 & $1.055-1.177$ & [0.001* \\
\hline \multicolumn{2}{|l|}{ TPB_V50 } & 1.142 & $1.069-1.221$ & {$[0.001 *$} \\
\hline
\end{tabular}

Abbreviation: BMI, body mass index; EBRT, pelvic external-beam radiotherapy; TPB_V10, TPB_V20, TPB_V30, TPB_V40, TPB_V50冈the total pelvic bones of volume receiving $10,20,30,40,50 \mathrm{~Gy}$; TP, paclitaxel + cisplatin. Bold indicates the significant values $\left({ }^{\star} p<0.05\right)$.

Subsequently, multivariate analysis was performed with all the significant factors in the univariate analysis. Results of this multivariate analysis demonstrated that the chemotherapy regimens and TPB_V20 were independent factors. Patients who received TP chemotherapy regimen were more likely to develop Grade $\geq 3 \mathrm{HT}$ than those who received cisplatin or carboplatin ( $76.2 \%$ vs. $58.6 \%$ vs. $45.2 \%)$. We also observed that the patient with increased TPB_V20 were more likely to develop Grade $\geq 3 \mathrm{HT}$ (OR 1.154; SE 0.69; $p=0.034 ; 95 \% \mathrm{Cl} 1.008-1.321$ ). Hosmer-Lemeshow test indicated that the models fitted well $(\chi 2=8.412, p$-value $=3.94 \bowtie 0.05)$. Detailed results are given in Figure 4 . 
The ROC curve for Grade $\geq 3$ hematologic toxicity versus TPB_V20 was analyzed to determine the optimal thresholds for dosimetric planning. The optimal TPB_V20 cut-off value identified by ROC curves followed by Youden test was 71\% (AUC $=0.788 ; 95 \% \mathrm{Cl}, 0.722-0.845 ; \mathrm{p}$-value冈0.001). Figure 5. Patients who received TPB_V20 $\geq 71 \%$ were more likely to develop Grade $\geq 3 \mathrm{HT}(84.1 \%$ vs. $38.5 \%, \mathrm{p}<0.001)$. The specificity and sensitivity for this threshold were $78.1 \%$ and $71.2 \%$, respectively. The positive and negative predictive values for TPB_V20 $\geq 71 \%$ were $83.1 \%$ (95\% Cl $77.3 \%-87.7 \%)$ and $64.1 \%(95 \% \mathrm{Cl} \times 56.7 \%-$ $70.8 \%)$, respectively, with a relative risk of $3.86(95 \% \mathrm{CI} \otimes 2.66-5.6)$.

\section{Discussion}

Acute hematological toxicity $\mathbb{\mathrm { HTT }} \mathbb{\mathrm { i }}$ is a common toxicity in cervical cancer patients who received concurrent chemoradiotherapy (CCRT), especially Grade $\geq 3 \mathrm{HT}$, which may reduce CCRT doses or termination of CCRT altogether冈and ultimately affect the therapeutic efficacy ${ }^{[5]}$. To our knowledge, this is the first report to explore the predictive value of $A M C$ in cervical cancer patients undergoing CCRT. In the present study, we observed that the trend of the AMC was consistent with the WBC/ANC during CCRT. The decrease and increase of $A M C$ occurred before the $A N C / W B C$, and there is a clear correlation between the nadir of AMC and the severity of neutropenia/leukopenia. In addition, the result of our study has provided quantitative evidence of an association between TPB_V20 and acute Grade $\geq 3$ HT.

Multiple studies have established that treatment-time prolongation negatively impacted the diseasespecific survival and local control rat in patients with cervical cancer underwent CCRT ${ }^{[10-12]}$. However, acute HT was responsible for the greater part of treatment-time prolongation囚especially with Grade $\geq 3$ neutropenia/leukopenia. Those patients are more likely to develop life-threatening infections or febrile neutropenia $₫$ which could reduce CCRT doses or terminate CCRT altogether囚and ultimately affect the therapeutic efficacy. The recommendation from the ASCO indicated that the granulocyte colony-stimulating factor(G-CSF) can be used for preventing febrile neutropenia in high-risk patients ${ }^{[13]}$. However, even if these patients are given G-CSF, their treatment time and cost will also be increased. In current clinical practice, especially the risk assessment, control, and prevention of lifethreatening infection or febrile neutropenia were not standardized. These situations suggest that minimizing $\mathrm{HT}$ is particularly important when introducing more intensive chemotherapy regimens for cervical cancer, and research on HT should be strengthened.

Previous studies have demonstrated that there was a statistically strong correlations between AMC and ANC during chemotherapy ${ }^{[14-19]}$. Kondo et al. showed that AMC $\otimes 150 /$ microl on days 6 to 8 might be a predictor of grade 3 or higher neutropenia during advanced lung cancer chemotherapy at 3- or 4-week intervals ${ }^{[14]}$. Subsequently, Oshita $\mathrm{F}$ et al. compared a group of 60 patients with unresectable lung cancer which were randomly assigned to prophylactic administration of G-CSF when monocytopenia appears \group A囚or administration of G-CSF when neutropenia ( $<1,000 /$ microl) or leukopenia ( $<2,000 /$ microl) appeared after chemotherapy $₫$ group $B \rrbracket^{[15]}$. The results showed that prophylactic administration of G-CSF can significantly shorten the duration ( $1.4 \pm 1.7$ days vs. $2.9 \pm 1.9$ days, $p=0.004)$ and frequency $(48 \%$ 
vs. $83 \%, p=0.002$ ) of grade 3 neutropenia ${ }^{[15]}$. Also, in another study of 75 patients with advanced lung cancer undergoing chemotherapy, results of this study showed that the number of days that elapsed between the first day of chemotherapy and the median AMC nadir was shorter than that for AMC nadir (6 days vs. 12 days, $P<0.001$ ); AMC at the nadir of $100 / \mu \mathrm{L}$ predicted an $\mathrm{ANC}$ at nadir $<1000 / \mu \mathrm{L}$ with a sensitivity of $83 \%$ and a specificity of $56 \%{ }^{[16]}$. Similar results were reported in a study that the changing trend of AMC was the same as that of ANC. The initial decrease / reaching the nadir / final increase days of AMC were significantly less than those of $A N C$, respectively ${ }^{[17]}$. Two other studies also demonstrated the association between the ANC and the AMC in patients receiving chemotherapy ${ }^{[18,19]}$.

Our retrospective study evaluated the correlation between AMC and ANC/WBC and the predictive value of AMC for acute leukopenia/neutropenia during cervical cancer patients undergoing CCRT. The present study results show that the decrease and increase of AMC usually occurred before the ANC and WBC\and the changing tendency of AMC had some predictive value to the changing trend of WBC and ANC. We also obtained that the nadir of AMC was positively correlated with the nadir of WBC / ANC. In fact, during hematopoiesis, both neutrophils and monocytes are differentiated from the granulocyte-macrophage colony-forming cell, providing a good explanation for a positive correlation between AMC and ANC nadirs during concurrent radiochemotherapy. Thus, based on the findings of previous studies and this study, it could be stated that the increase or decrease of $A M C$ can be used as an effective predictor for the timing and severity of the ANC/WBC nadirs and prophylactic administration of G-CSF.

Additionally, all the blood cell counts of patients were decreased to various degrees throughout the study period. The WBC, ANC, HGB, AMC and PLT counts decreased by $64.72 \%, 64.86 \%, 16.00 \%, 50.00 \%$, $53.44 \%$,respectively. The percentage of patients with Grade 3 or Grade 4 neutropenia, leukopenia, thrombocytopenia, and anemia during concurrent chemoradiotherapy was $52.17 \%, 30.43 \%, 6.52 \%$, and $22.83 \%$, respectively. Overall, 111 patients $₫ 60.33 \% \bigotimes$ did experience any Grade $3+\mathrm{HT}$. We found that in most patients, white blood cells and neutrophil counts decreased significantly during CCRT. Current treatment options for the management of stage IB- IVA cervical cancer is weekly cisplatin plus radiotherapy. However, in some studies, different results were obtained. A meta-analysis by Petrelli $F$ et al. revealed that platinum-based doublet chemotherapy plus concurrent radiotherapy can improve the overall survival and disease-free survival compared to weekly cisplatin plus radiotherapy. Still, severe HT was largely underreported in the included publications ${ }^{[20]}$. In our study, weekly cisplatin and weekly carboplatin were significantly more effective in reducing $\geq 3$ hematologic toxicity than the TP regimen ( $58.6 \%$ vs. $45.2 \%$ vs. $76.2 \%$ ). Therefore, it is essential to select the best chemotherapy regimens for individual patients that may decrease the chemotherapy-related hematologic toxicity.

Several studies had analyzed the relationship between pelvic dosimetry parameters and $\mathrm{HT}$ in patients who undergo pelvic radiotherapy with respect to radiation doses. Mell et al. analyzed 37 patients withe cervical cancer who received CCRT and found the risk of Grade 2 or grater leukopenia and neutropenia developing increases by a factor (odds ratio) of 2.09 if the TPB_V10 exceeds $90 \%{ }^{[9]}$. Rose et al. found that HT increases with increasing the total pelvic bone volume irradiated, and efforts to keep TPB_V10< 
$95 \%$ and TPB_V20 $<76 \%$ may avoid Grade $3+$ leukopenia ${ }^{[21]}$. Additionally, a recent study demonstrated that lower pelvis $\varangle \mathrm{V} 5>95 \%$ or $\mathrm{V} 20>45 \%$ ) $\mathbb{T} \mathrm{TPB} \_\mathrm{V} 20 \geq 65 \%$, and the mean dose of iliac crests $>31$ Gy was correlated most significantly with Grade $4 \mathrm{HT}^{[8]}$. For rectal cancer patients, Wan et al. demonstrated lumbosacral spine V40 was correlated most significantly with grade $\geq 2 \mathrm{HT}$ and recommended dose constraints to the lumbosacral spine was $\mathrm{V} 40<60 \%{ }^{[22]}$. For patients with anal cancer, Franco et al. found that a higher TPB_V20 was associated with lower WBC nadir, and patients with lumbosacral spine V40 $<41 \%$ may reduce Grade 3+ HT. In summary, no optimal bone marrow dose/volume constraints standard has been proposed until now.

In several studies, the external contour of all the pelvis bones was delineated as TBP. Then the TBP was divided into three subsites: lumbosacral spine, lower pelvis, and ilium, and the importance of these particular regions was stated ${ }^{[8,22,26]}$. However, in our study, all the external contours of the bones within the PTV coverage were delineated as a proxy for the total pelvic bones(TPB) to ensure repeatability and to avoid multiple collinearities in the multivariate analysis. Our results are consistent with those from other studies in those patients with higher volumes of TPB receiving low-dose radiation had higher rates of Grade $\geq 3 \mathrm{HT}^{[21,29]}$. Patients who received TPB_V20 $\geq 71 \%$ were more likely to develop Grade $\geq 3$ $\mathrm{HT}(84.1 \%$ vs. $38.5 \%, \mathrm{p}<0.001)$. The specificity and sensitivity for this threshold were $78.1 \%$ and $71.2 \%$, respectively. The association with the low-dose dosimetric parameter(TPB_V20) was consistent with the bone marrow's known radiosensitivity.

However, certain limitations remain in our study. First of al, this was a single-center retrospective study with a relatively small number of patients, leading to selection bias. Furthermore, the dosage and duration of G-CSF treatment varied according to the different conditions of patients during the treatment course, which may have a certain impact on the changing trend of AMC, ANC, and WBC. Finally, we contoured the external contours of the bones as opposed to the actual proliferating active bone. Despite the limitations outlined above, the present study still highlights the predictive value of dosimetric parameters and AMC for HT in cervical cancer patients underwent CCRT.

\section{Conclusion}

Based on the findings of our study, the following conclusions have been drawn. The changing trend of AMC can be used as an effective predictor for the timing and severity of the ANC/WBC nadirs and prophylactic administration of G-CSF. In addition, according to the multivariate logistic regression analysis, the chemotherapy regimen and TPB_V20 were independent risk factors for the development of Grade $\geq 3$ hematologic toxicity. Maintain TPB_V20 $<71 \%$ and selecting single-agent cisplatin or carboplatin could significantly reduce Grade $\geq 3$ hematologic toxicity in cervical cancer patients undergoing concurrent chemoradiotherapy. However, the predictive value of dosimetric parameters and AMC for acute hematologic toxicity remains further investigated.

\section{Abbreviations}


VMAT: volumetric-modulated arc therapy; CC: cervical cancer; CCRT: concurrent chemoradiotherapy; HT, hematological toxicity; AMC: absolute monocyte count; ANC: absolute neutrophil count; WBC: white blood cells; IMRT: intensity-modulated radiation therapy; TOMO: helical tomography radiotherapy; 3D-CRT: conventional three-dimensional conformal radiotherapy; OARs: organs at risk; TPB: total pelvic bones; LP: lower pelvis; LS: lumbosacral spine; Q1, 25th percentile; Q3, 75th percentile; SD, standard deviation; G-CSF, granulocyte colony-stimulating factor;

\section{Declarations}

\section{Acknowledgements}

Not applicable.

\section{Authors' contributions}

Ning Li and Xiaoyong Xiang was responsible for the primary concept and the design of the study; Xiaoyong Xiang, Zhen Ding, and Qi Zeng performed the data capture and analysis; Xiaoyong Xiang, Zhen Ding, and Ning Li drafted the manuscript; All the external contours of the bones were delineated by Xiaoyong Xiang and subsequently reviewed by Ning Li; Lingling Feng, Chunyan Qiu, Dongjie Chen, and Jiawei Lu provided study materials or patients; All authors revised the manuscript. All authors have read and approved the final manuscript.

\section{Funding}

Supported by the Sanming Project of Medicine in Shenzhen『No.SZSM201612063】and the Shenzhen Key Medical Discipline Construction Fund囚No.SZXK013》.

\section{Availability of data and materials}

The datasets used during the current study are available from the corresponding author on reasonable request.

\section{Ethics approval and consent to participate}

The study was approved by the ethics committee of The Cancer Hospital \& Shenzhen Hospital.

\section{Consent for publication}

Not applicable.

\section{Competing interests}

None of the authors have any conflicts of interest to declare.

\section{Author details}


3. Department of Radiation Oncology, National Cancer Center/National Clinical Research Center for Cancer/Cancer Hospital \& Shenzhen Hospital, Chinese Academy of Medical Sciences and Peking Union Medical College, Shenzhen, 518116, China

4. Department of Radiation Oncology, National Cancer Center/National Clinical Research Center for Cancer/Cancer Hospital, Chinese Academy of Medical Sciences and Peking Union Medical College, Beijing, 100021, China

\section{References}

1. Sung H, Ferlay J, Siegel R L, et al. Global cancer statistics 2020: GLOBOCAN estimates of incidence and mortality worldwide for 36 cancers in 185 countries[J]. CA: a cancer journal for clinicians, 2021.DOI:10.3322/caac.21660.

2. Huang $H$, Feng $Y L$, Wan T, et al. Effectiveness of Sequential Chemoradiation vs Concurrent Chemoradiation or Radiation Alone in Adjuvant Treatment After Hysterectomy for Cervical Cancer: The STARS Phase 3 Randomized Clinical Trial[J]. JAMA Oncol, 2021,7(3):361369.DOI:10.1001/jamaoncol.2020.7168.

3. Shrivastava S, Mahantshetty U, Engineer R, et al. Cisplatin Chemoradiotherapy vs Radiotherapy in FIGO Stage IIIB Squamous Cell Carcinoma of the Uterine Cervix[J]. JAMA Oncology, 2018,4(4):506.DOI:10.1001/jamaoncol.2017.5179.

4. Cohen P A, Jhingran A, Oaknin A, et al. Cervical cancer[J]. Lancet, 2019,393(10167):169182.DOI:10.1016/S0140-6736(18)32470-X.

5. Crawford J, Dale D C, Kuderer N M, et al. Risk and timing of neutropenic events in adult cancer patients receiving chemotherapy: the results of a prospective nationwide study of oncology practice[J]. Journal of the National Comprehensive Cancer Network, 2008,6(2):109.

6. Hayman J A, Callahan J W, Herschtal A, et al. Distribution of Proliferating Bone Marrow in Adult Cancer Patients Determined Using FLT-PET Imaging[J]. International Journal of Radiation Oncology*Biology*Physics, 2011,79(3):847-852.DOI:10.1016/j.jjrobp.2009.11.040.

7. Yu D, Bai Y, Feng Y, et al. Which Bone Marrow Sparing Strategy and Radiotherapy Technology Is Most Beneficial in Bone Marrow-Sparing Intensity Modulated Radiation Therapy for Patients With Cervical Cancer?[J]. Frontiers in Oncology, 2020,10.DOI:10.3389/fonc.2020.554241.

8. Kumar T, Schernberg A, Busato F, et al. $\langle p>$ Correlation between pelvic bone marrow radiation dose and acute hematological toxicity in cervical cancer patients treated with concurrent chemoradiation</p>[J]. 2019,Volume 11:6285-6297.DOI:10.2147/CMAR.S195989.

9. Mell L K, Kochanski J D, Roeske J C, et al. Dosimetric predictors of acute hematologic toxicity in cervical cancer patients treated with concurrent cisplatin and intensity-modulated pelvic radiotherapy [J]. International Journal of Radiation Oncology*Biology*Physics, 2006,66(5):13561365.DOI:10.1016/j.jijrobp.2006.03.018. 
10. Lin S M, Ku H Y, Chang T C, et al. The prognostic impact of overall treatment time on disease outcome in uterine cervical cancer patients treated primarily with concomitant chemoradiotherapy: a nationwide Taiwanese cohort study[J]. Oncotarget, 2017,8(49):85203-

85213.DOI:10.18632/oncotarget.19617.

11. Chen S, Liang J, Yang S, et al. The adverse effect of treatment prolongation in cervical cancer byhighdose-rate intracavitary brachytherapy[J]. Radiotherapy and Oncology, 2003,67(1):69-

76.DOI:10.1016/S0167-8140(02)00439-5.

12. Mazeron R, Castelnau-Marchand P, Dumas I, et al. Impact of treatment time and dose escalation on local control in locally advanced cervical cancer treated by chemoradiation and image-guided pulsed-dose rate adaptive brachytherapy[J]. Radiotherapy and Oncology, 2015,114(2):257263.DOI:10.1016/j.radonc.2014.11.045.

13. Mell L K, Kochanski J D, Roeske J C, et al. Dosimetric predictors of acute hematologic toxicity in cervical cancer patients treated with concurrent cisplatin and intensity-modulated pelvic radiotherapy [J]. International Journal of Radiation Oncology*Biology*Physics, 2006,66(5):13561365.DOI:10.1016/j.ijrobp.2006.03.018.

14. Kondo M, Oshita F, Kato Y, et al. Early monocytopenia after chemotherapy as a risk factor for neutropenia[J]. Am J Clin Oncol. 1999;22(1):103-105. DOI:10.1097/00000421-199902000-00025.

15. Oshita F, Yamada K, Nomura I, et al. Prophylactic administration of granulocyte colony-stimulating factor when monocytopenia appears lessens neutropenia caused by chemotherapy for lung cancer[J]. Am J Clin Oncol. 2000 Jun;23(3):278-82. DOI: 10.1097/00000421-200006000-00014.

16. Moriyama $\mathrm{Y}$, Horita $\mathrm{N}$, Kudo $\mathrm{M}$, et al. Monocyte nadir is a possible indicator for neutrophil nadir during lung cancer chemotherapy[J]. Clin Respir J. 2017;11(4):453-458. DOI:10.1111/crj.12358

17. Ouyang W, Liu Y, Deng D, et al. The change in peripheral blood monocyte count: A predictor to make the management of chemotherapy-induced neutropenia[J]. J Cancer Res Ther. 2018;14(Supplement):S565-S570. DOI:10.4103/0973-1482.177502.

18. Sato I, Nakaya N, Shimasaki T, et al. Prediction of docetaxel monotherapy-induced neutropenia based on the monocyte percentage[J]. Oncol Lett. 2012;3(4):860-864. doi:10.3892/ol.2012.556

19. Shimanuki M, Imanishi Y, Sato Y, et al. Pretreatment monocyte counts and neutrophil counts predict the risk for febrile neutropenia in patients undergoing TPF chemotherapy for head and neck squamous cell carcinoma[J]. Oncotarget. 2018;9(27):18970-18984. Published 2018 Apr 10. DOI:10.18632/oncotarget.24863.

20. Petrelli F, De Stefani A, Raspagliesi F, et al. radiotherapy with concurrent cisplatin-based doublet or weekly cisplatin for cervical cancer: a systematic review and meta-analysis[J]. Gynecol Oncol. 2014;134(1):166-171. DOI:10.1016/j.ygyno.2014.04.049.

21. Rose B S, Aydogan B, Liang Y, et al. Normal Tissue Complication Probability Modeling of Acute Hematologic Toxicity in Cervical Cancer Patients Treated With Chemoradiotherapy[J]. International Journal of Radiation Oncology*Biology*Physics, 2011,79(3):800-

807.DOI:10.1016/j.ijrobp.2009.11.010.

Page $15 / 19$ 
22. Wan J, Liu K, Li K, et al. Can dosimetric parameters predict acute hematologic toxicity in rectal cancer patients treated with intensity-modulated pelvic radiotherapy?[J]. Radiation Oncology, 2015,10(1).DOI:10.1186/s13014-015-0454-0.

23. Klopp A H, Moughan J, Portelance L, et al. Hematologic Toxicity in RTOG 0418: A Phase 2 Study of Postoperative IMRT for Gynecologic Cancer[J]. International Journal of Radiation Oncology*Biology*Physics, 2013,86(1):83-90.DOI:10.1016/j.ijrobp.2013.01.017.

24. Rose B, Mitra D, Hong T S, et al. irradiation of anatomically defined pelvic subsites and acute hematologic toxicity in anal cancer patients undergoing chemoradiation[J]. Pract Radiat Oncol, 2017,7(5):e291-e297.DOI:10.1016/j.prro.2017.03.008.

25. Lee A Y, Golden D W, Bazan J G, et al. Hematologic Nadirs During Chemoradiation for Anal Cancer: Temporal Characterization and Dosimetric Predictors[J]. Int J Radiat Oncol Biol Phys, 2017,97(2):306-312.DOI:10.1016/j.jirobp.2016.10.010.

26. Franco $P$, Ragona $R$, Arcadipane $F$, et al. Dosimetric predictors of acute hematologic toxicity during concurrent intensity-modulated radiotherapy and chemotherapy for anal cancer[J]. Clinical and Translational Oncology, 2017,19(1):67-75.DOI:10.1007/s12094-016-1504-2.

27. Chang Y, Yang Z, Li G, et al. Correlations Between Radiation Dose in Bone Marrow and Hematological Toxicity in Patients With Cervical Cancer: A Comparison of 3DCRT, IMRT, and RapidARC[J]. International Journal of Gynecologic Cancer, 2016,26(4):770-776.DOI:10.1097.

28. Bazan JG, Luxton G, Mok EC, et al. Chang DT. Normal tissue complication probability modeling of acute hematologic toxicity in patients treated with intensity-modulated radiation therapy for squamous cell carcinoma of the anal canal[J]. Int J Radiat Oncol Biol Phys. 2012;84(3):700-706. DOI:10.1016/j.ijrobp.2011.12.072.

29. Albuquerque K, Giangreco D, Morrison C, et al. Radiation-related predictors of hematologic toxicity after concurrent chemoradiation for cervical cancer and implications for bone marrow-sparing pelvic IMRT[J]. Int J Radiat Oncol Biol Phys. 2011;79(4):1043-1047. DOI:10.1016/j.ijrobp.2009.12.025.

\section{Figures}

\section{Figure 1}

Typical figures were showing contours for the total pelvic bone(TPB)[9]. 
(A) $\quad$ WBC

ANC

(C)

AMC

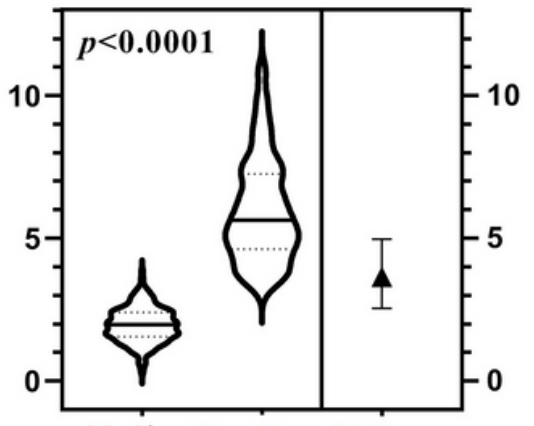

Nadir Baseline Difference

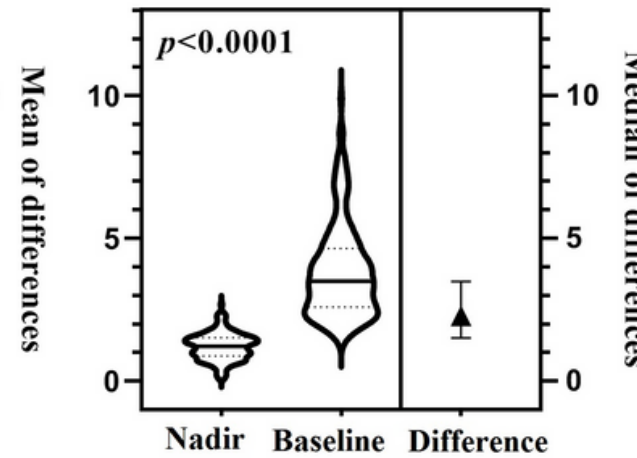

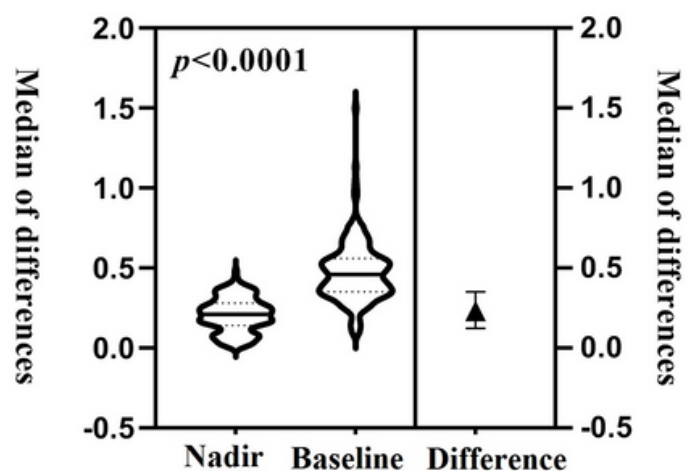

(E)

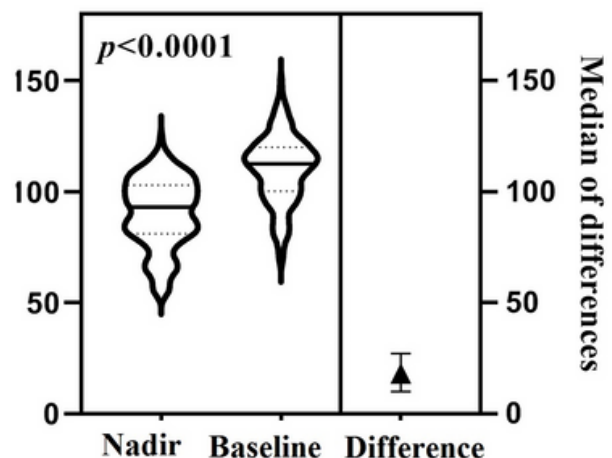

HGB

Figure 2

(D)

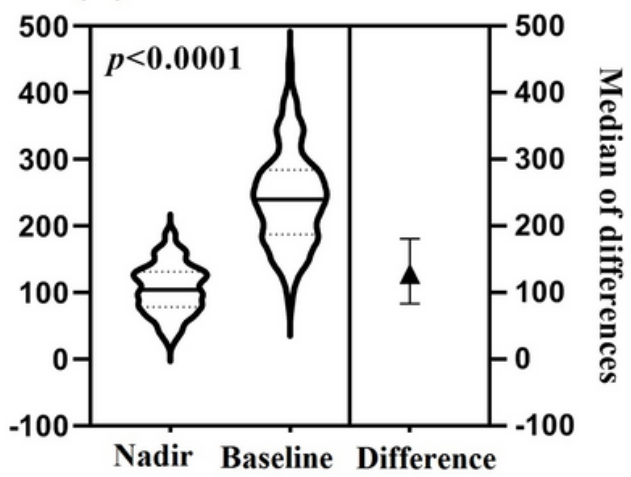

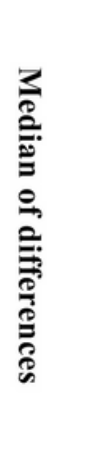

Descriptive statistics of hematological baselines and nadirs.

(A)

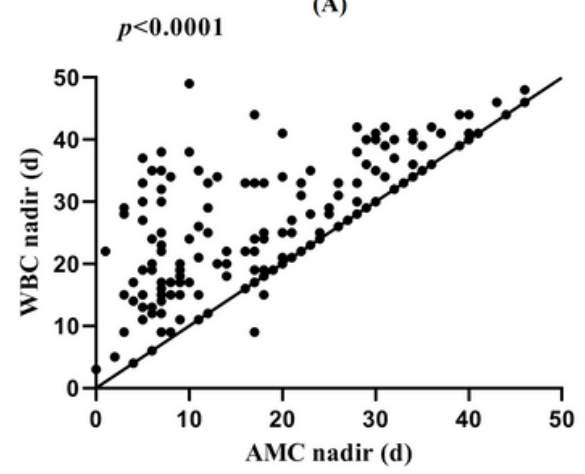

(D)

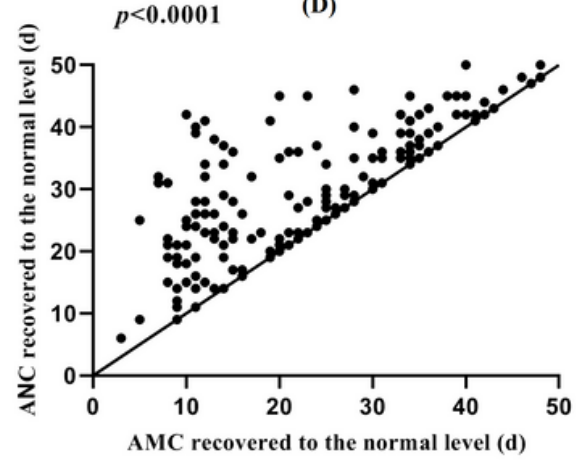

(B)
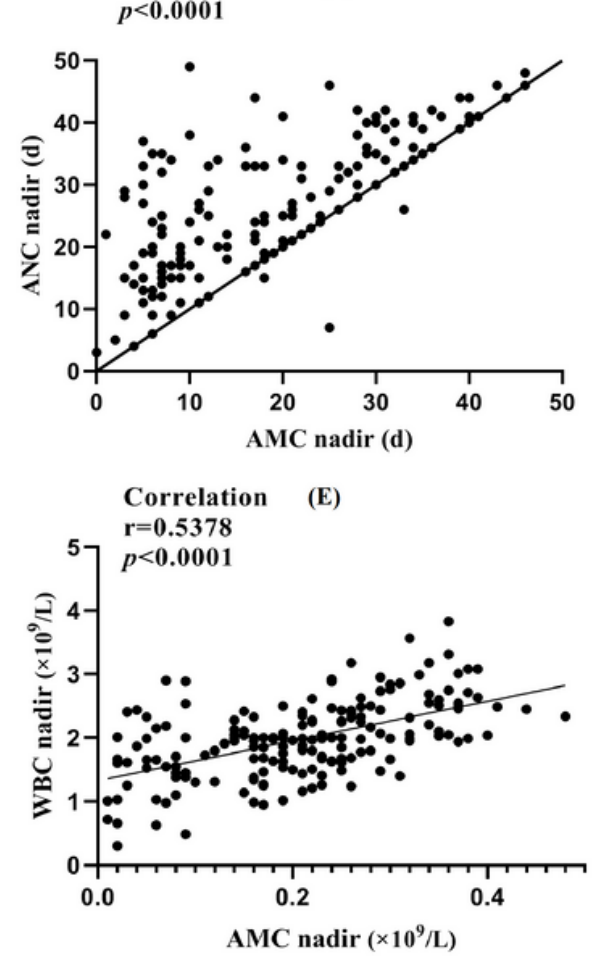

(C)

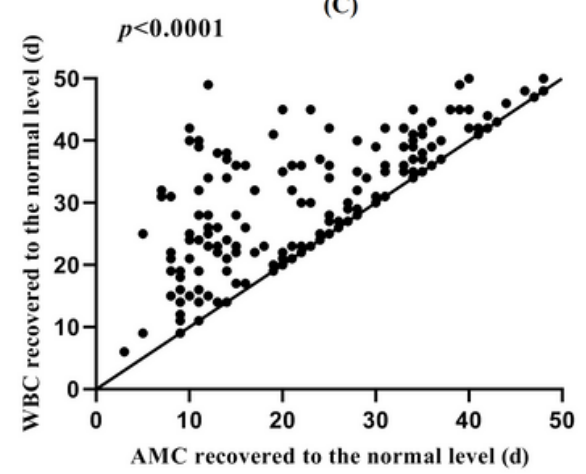

(F)

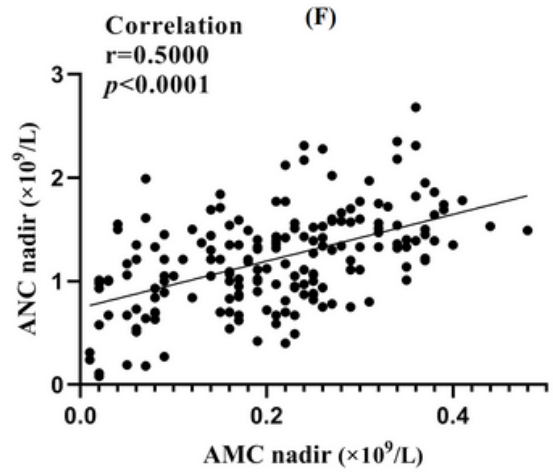


Figure 3

The changing tendency and correlation between AMC and ANC/WBC.

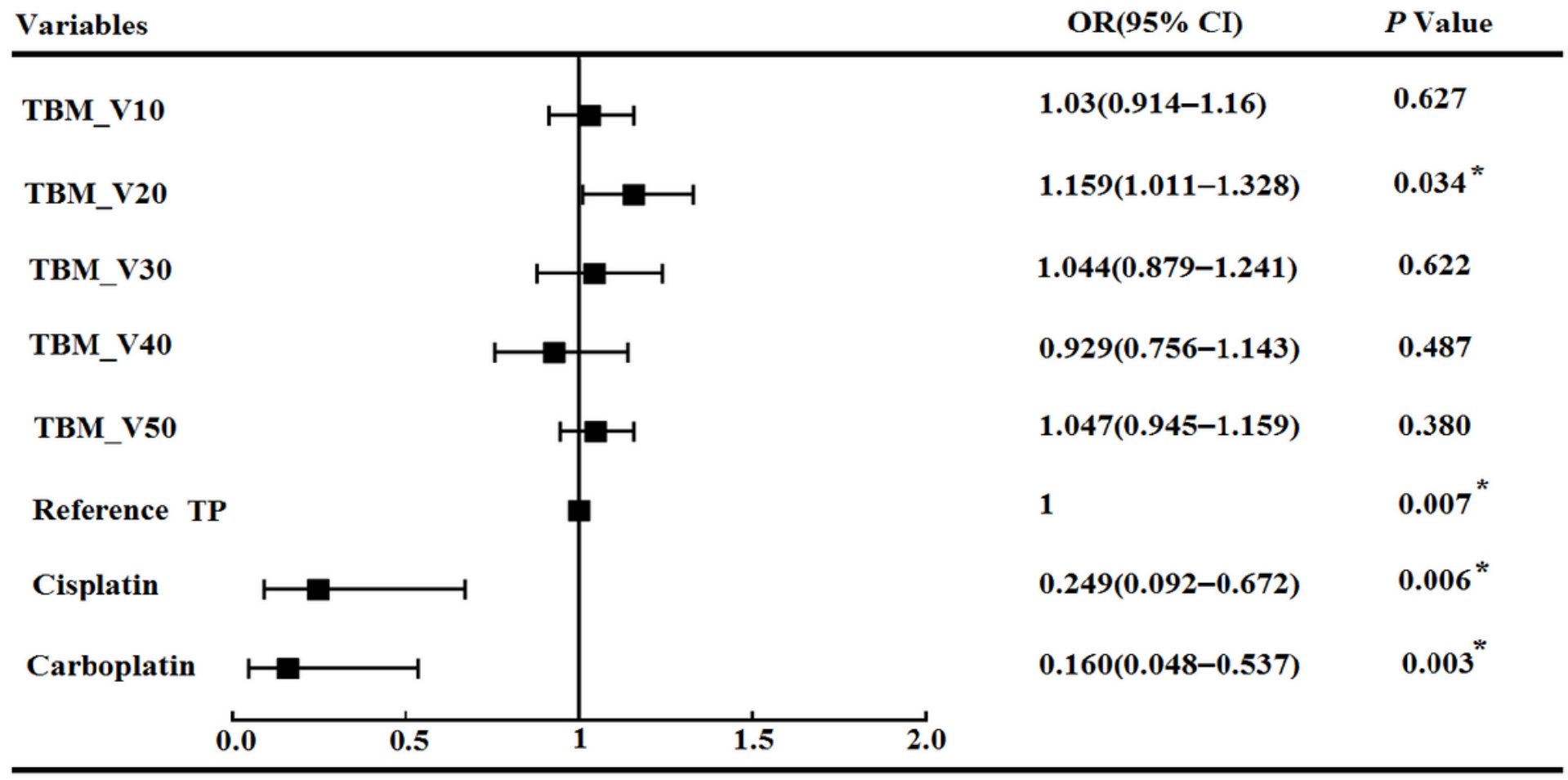

Figure 4

The results of multivariate binary logistic regression analysis for any Grade $\geq 3 \mathrm{HT}$ in patients. 


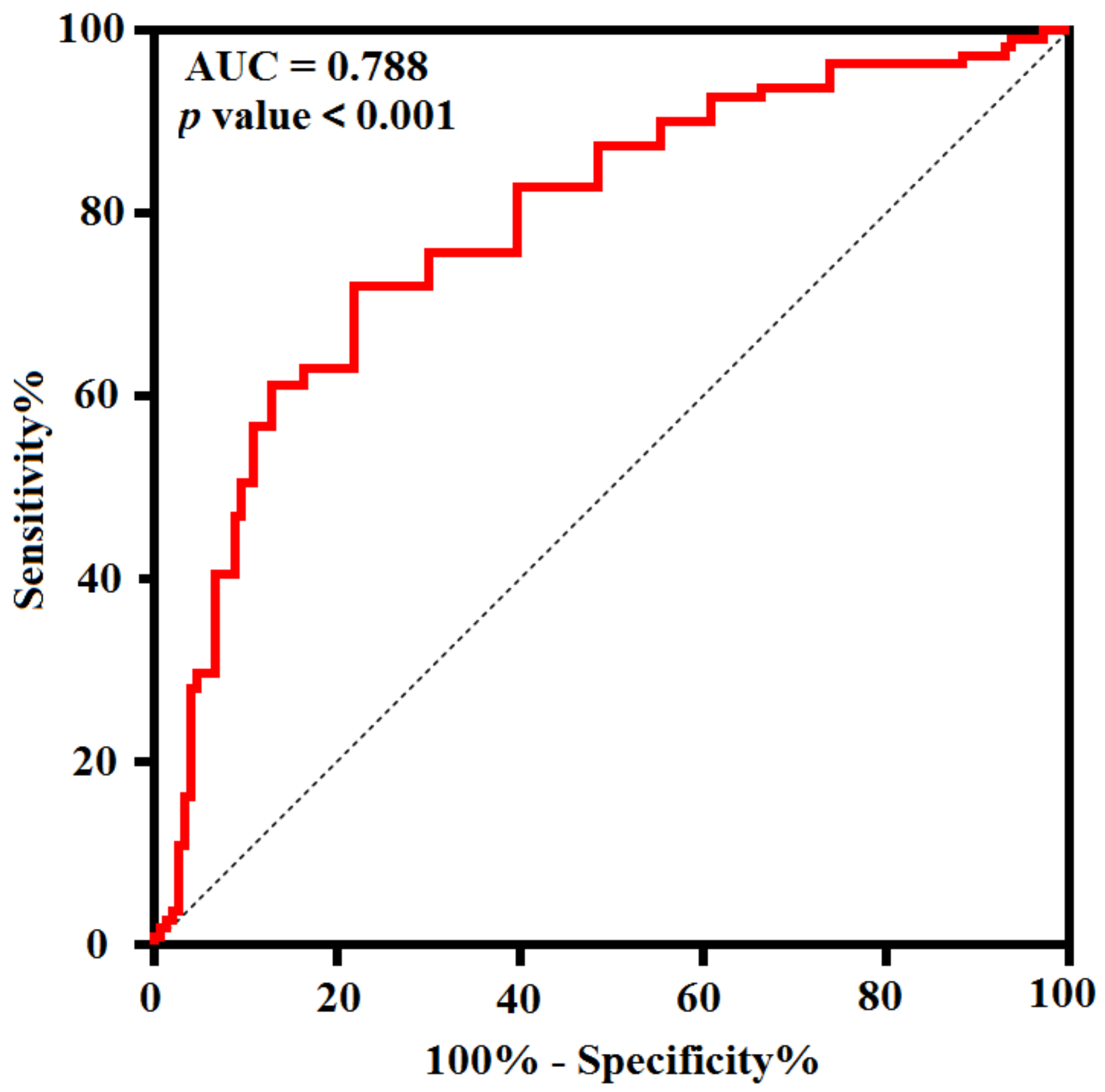

Figure 5

Receiver operating characteristic (ROC) curves for any Grade $\geq 3$ hematologic toxicity as a function of TPB_V20. 